\title{
Barriers for the injured indigenous person to return to work
}

\author{
Howard Flavell \\ Northern Rehabilitation Network, Tiwi NT 0810, Australia. Howard.Flavell@nt.gov.au
}

\begin{abstract}
Aboriginal and Torres Strait Islander people carry a high disease burden with a consequent reduced life expectancy. There are reduced opportunities for conventional employment with further difficulties if the person has a disability. Nevertheless meaningful participation in community activities can occur. This paper will explore these issues giving examples of how this can be facilitated.
\end{abstract}

